\title{
Gout and risk of chronic kidney disease and nephrolithiasis: meta-analysis of observational studies
}

\author{
Matthew J Roughley ${ }^{1}$, John Belcher ${ }^{2}$, Christian D Mallen ${ }^{3}$ and Edward Roddy ${ }^{3 *}$
}

\begin{abstract}
Introduction: To determine the prevalence of chronic kidney disease and nephrolithiasis in people with gout, and the association between gout and prevalent or incident chronic kidney disease and nephrolithiasis.

Methods: Systematic review and meta-analysis of epidemiological studies. Data sources; MEDLINE, EMBASE and CINAHL databases, hand-searched reference lists, citation history and contact with authors. Eligibility criteria: cohort, case-control or cross-sectional studies which examined the occurrence of chronic kidney disease or nephrolithiasis amongst adults with gout (with or without a non-gout comparator group) in primary care or general population samples. Prevalence and risk estimate meta-analyses were performed using a random-effects model.

Results: Seventeen studies were included in the meta-analysis (chronic kidney disease $n=7$, nephrolithiasis $n=8$, both $n=2$ ). Pooled prevalence estimates of chronic kidney disease stage $\geq 3$ and self-reported lifetime nephrolithiasis in people with gout were $24 \%$ (95\% confidence interval 19\% to $28 \%$ ) and $14 \%$ (95\% Cl 12\% to 17\%) respectively. Gout was associated with both chronic kidney disease (pooled adjusted odds ratio $2.41,95 \%$ confidence interval 1.86 to 3.11 ) and self-reported lifetime nephrolithiasis $(1.77,1.43$ to 2.19$)$.

Conclusions: Chronic kidney disease and nephrolithiasis are commonly found amongst patients with gout. Gout is independently associated with both chronic kidney disease and nephrolithiasis. Patients with gout should be actively screened for chronic kidney disease and its consequences.
\end{abstract}

\section{Introduction}

Gout is the most prevalent inflammatory arthritis, affecting $2.4 \%$ of adults in the UK [1]. Gout is associated with considerable co-morbidity including hypertension, diabetes mellitus, obesity, metabolic syndrome, and vascular disease [2]. Associations between gout and renal disease and nephrolithiasis have long been recognised, yet early studies undertaken in specialist secondary care populations are likely to be unrepresentative of most patients with gout who are managed exclusively in primary care settings [3-6]. To the best of our knowledge, no previous systematic reviews examining this association have been performed.

Chronic kidney disease (CKD) stages 3 to 5 (glomerular filtration rate $<60 \mathrm{ml} /$ minute $/ 1.73 \mathrm{~m}^{2}$ ) affects $8.5 \%$ of the UK population, is more common in females than

\footnotetext{
*Correspondence: e.roddy@keele.ac.uk

${ }^{3}$ Arthritis Research UK Primary Care Centre, Keele University, Keele, Staffordshire ST5 5BG, UK

Full list of author information is available at the end of the article
}

males (10.6\% vs. $5.8 \%)$, and is associated with both allcause mortality and cardiovascular disease $[7,8]$. If left untreated, CKD may progress to end-stage renal disease (ESRD) requiring expensive renal replacement therapy (RRT) [9]. CKD is an independent risk factor for the development of gout $[10,11]$ yet there are several reasons to explain why gout may predispose to renal disease, including hyperuricaemia, chronic inflammation, co-morbid hypertension and diabetes mellitus, and use of nonsteroidal antiinflammatory drugs. The management of gout in patients with renal disease is challenging because nonsteroidal antiinflammatory drugs and colchicine should be used with caution and lower doses of allopurinol are required [12].

The lifetime prevalence of nephrolithiasis is approximately $8.8 \%$ [13]. Kidney stones most commonly present as renal colic but can be complicated by infection, renal tract obstruction requiring surgical intervention, and renal failure [14]. Gout is associated with lower urinary $\mathrm{pH}$ theoretically predisposing to the formation of both 
uric acid and other stones [5,15]. Diabetes mellitus, obesity, and hypertension are also known to be independent risk factors for nephrolithiasis [16-18].

The objectives of this systematic review and metaanalysis of epidemiological studies were to quantify the prevalence of CKD and nephrolithiasis in gout, and to determine whether gout is associated with prevalent or incident CKD and nephrolithiasis in the general population.

\section{Methods}

\section{Data sources and searches}

A systematic literature search was undertaken by two coinvestigators (MJR and ER) in the MEDLINE, EMBASE, and CINAHL databases using the National Health Service's Healthcare Databases Advanced Search from inception date to November 2013 for epidemiological studies examining the association between gout and either CKD or nephrolithiasis. Search terms pertaining to gout were combined with terms describing the outcome (CKD or nephrolithiasis) and observational study design using the Boolean operator "AND". No unpublished data sources were searched. The references and citation history of all relevant studies were checked for additional data sources.

\section{Eligibility criteria}

Eligible studies were required to include adults with gout (with or without a nongout comparator group), describe the occurrence of CKD or nephrolithiasis amongst adults with gout (and nongout comparator group where included), be of an epidemiological design (cross-sectional, cohort, case-control), and have been undertaken in a primary care or general population sample. No geographical or language restrictions were imposed. Conference abstracts were not included.

\section{Study selection}

MJR and ER independently screened the title and abstract of all identified studies. Studies which could not be excluded on title/abstract review were retained for full-text review undertaken by the same reviewers. At both the title/abstract and full-text review stages, disagreement over study eligibility was resolved by consensus discussion between the reviewers. A third reviewer (CDM) was available where disagreement could not be resolved by consensus.

\section{Data extraction and quality assessment}

The following data were extracted independently by the same two reviewers from studies meeting the inclusion criteria: date and location of study, study population and size, study type, demographic characteristics (age and gender), method of ascertainment of gout and CKD/nephrolithiasis, prevalence and incidence of $\mathrm{CKD} /$ nephrolithiasis in gout and comparator populations, unadjusted and adjusted risk estimates (odds ratio, relative risk, hazards ratio) and corresponding 95\% confidence interval (CI) or standard error, and details of covariates included in multivariate models. Studies utilising any method of gout ascertainment (for example, crystal identification, classification criteria, clinical diagnosis) were included. Staging of CKD was defined according to the National Kidney Foundation classification (stage 1, glomerular filtration rate $\geq 90 \mathrm{ml} /$ minute $/ 1.73 \mathrm{~m}^{2}$; stage 2 , glomerular filtration rate 60 to $89 \mathrm{ml} /$ minute $/ 1.73 \mathrm{~m}^{2}$; stage 3 , glomerular filtration rate 30 to $59 \mathrm{ml} /$ minute $/ 1.73 \mathrm{~m}^{2}$; stage 4 , glomerular filtration rate 15 to $29 \mathrm{ml} /$ minute $/ 1.73 \mathrm{~m}^{2}$; stage 5 (ESRD), glomerular filtration rate $<15 \mathrm{ml} /$ minute $/ 1.73 \mathrm{~m}^{2}$ ) [19]. Assessment of methodological quality was performed independently by the same two reviewers using the Newcastle-Ottawa quality appraisal tool with additional items regarding the method of diagnosis of gout, CKD, and nephrolithiasis [20]. Disagreement over data extraction and assessment of methodological quality was resolved by consensus discussion between the reviewers. Authors were contacted to request additional information and data where necessary.

\section{Data analysis}

Estimates of point/lifetime prevalence of CKD/nephrolithiasis in adults with gout and, where possible, risk estimates of the association between gout and CKD/ nephrolithiasis were calculated. These estimates were derived from summary $2 \times 2$ tables, together with regression estimates arising from adjusted analyses using logistic and Cox regression.

To normalize the distribution, prevalence rates were transformed by means of the logit event rate:

$$
\operatorname{Lp}=\operatorname{Ln}(\mathrm{p} /(1-\mathrm{p}))
$$

with $\mathrm{p}$ being the prevalence rate, $\mathrm{Ln}$ the natural logarithm, and $L p$ the logit event rate. The sampling variance of each logit event rate, $\mathrm{V}(\mathrm{Lp})$, was calculated by means of:

$$
\mathrm{V}(\mathrm{Lp})=1 /(\mathrm{np})+1 /(\mathrm{n}(1-\mathrm{p}))
$$

with $\mathrm{n}$ being the sample size. Once the statistical analyses were carried out, the results were back transformed to prevalence rates to facilitate their interpretation.

Separate analyses were undertaken for CKD and nephrolithiasis. Where sufficient data were present in individual studies but risk estimates were not reported, these were calculated using the available data. Pooled estimates were calculated using a random effects model. The $95 \%$ CIs and forest plots were produced for all pooled estimates.

Heterogeneity was assessed visually with forest plots, and quantified numerically using the $I^{2}$ index [21] and Cochran's $Q$ test. The $I^{2}$ test describes the percentage of variation 
across studies due to heterogeneity rather than chance. Meta-analyses were performed using the metan command within STATA 12.1 (StataCorp, College Station, TX, USA).

\section{Results}

Study selection

The search identified 2,032 potentially relevant articles: after removal of duplicates, 1,419 remained (Figure 1). Following title/abstract review, 1,333 articles were excluded. Of the 86 articles remaining, 57 were excluded after full-text review and 29 met the eligibility criteria. Seventeen studies contained data suitable for pooling. Reasons for exclusion are shown in Figure 1.

\section{Study characteristics}

Twelve studies examined CKD [22-33], 15 studies examined nephrolithiasis [13,34-47], and two studies examined both CKD and nephrolithiasis [48,49] (Table 1). Nineteen cross-sectional studies $[13,22,25,27,29,30,32$, 34-38,40-42,46-49], one case-control study [26], and nine cohort studies $[23,24,28,31,33,39,43-45]$ were identified. Six of the nine cohort studies included relevant outcome data only at baseline and so were treated as cross-sectional studies [24,31,33,43-45].

Fourteen studies were performed in the USA $[13,23$, $25-27,32,34,38,39,41,43-45,48]$, four in the UK [36,37,40, 49], five in Taiwan $[24,28,30,31,33]$, and one study each in China [22], Denmark [35], France [29], Indonesia [42], Italy [46], and Japan [47]. One study restricted participation to people aged over 65 years [44] and three studies included only males $[35,39,47]$. Fourteen studies were undertaken in large healthcare databases [23-28,31,33, 40,41,43-46] whereas 15 studies used empirically collected data $[13,22,29,30,32,34-39,42,47-49]$.

\section{Assessment of methodological quality}

Only three of 29 studies based the diagnosis of gout on validated clinical classification criteria: two studies [39,42] used the American Rheumatism Association criteria [50], and one study [34] used the New York and Rome criteria $[51,52]$. No studies based the diagnosis of gout on crystal identification. Three studies reported combined outcomes for gout and hyperuricaemia $[26,30,47]$. Nine studies used biochemical measures to define CKD [22,24,25,27,29, $30,32,48,49]$, four studies examined ESRD requiring RRT $[23,26,28,31]$, whereas nine of 17 studies of nephrolithiasis defined outcome by self-report [13,34,35,38,39,42,47-49], six studies used medical record linkage [40,41,43-46], and two studies used a general practitioner completed questionnaire [36,37].

Participants were considered not representative of the typical community gout patient in eight studies due to including only patients prescribed urate-lowering therapy $[25,44,45]$, only males $[35,39,47]$, or only people with chronic gout [42] and one study being undertaken in a Veterans database which resulted in a predominantly male (99\%) older population (mean age 72 years) [27]. All studies featuring a nongout comparator group had drawn this from the same community as the exposed cohort. Response and data usage rates were frequently not reported $[13,26,38,41-45,48]$, Two surveys reported response rates under $60 \%[30,49]$ and two database studies based in a
2032 records identified through database searching
11 additional records identified

through other sources
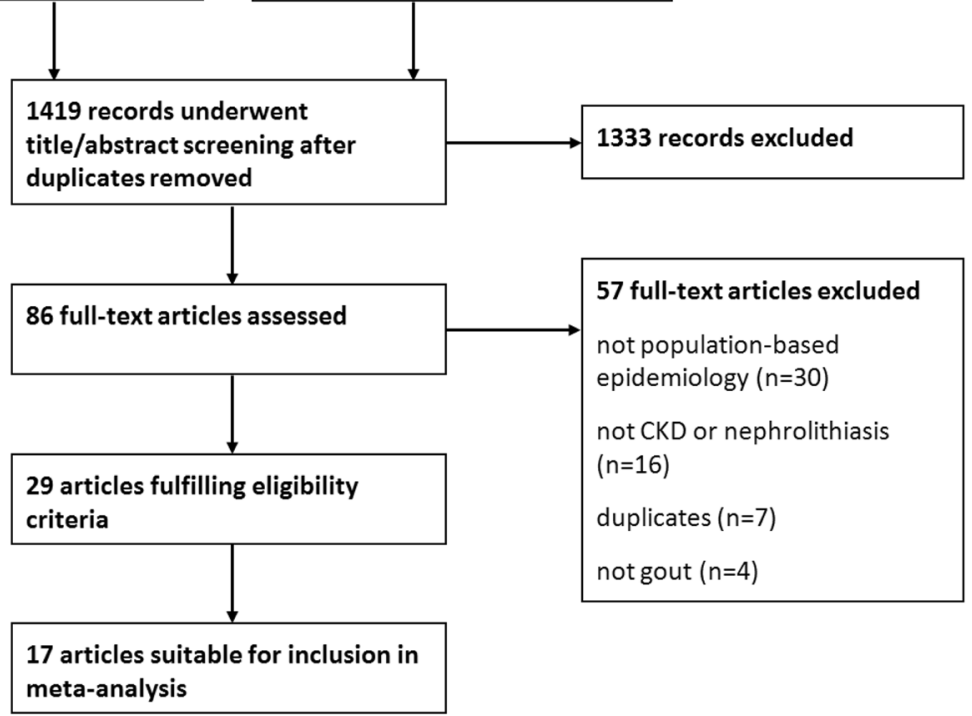

57 full-text articles excluded

not population-based

epidemiology $(n=30)$

not CKD or nephrolithiasis

$(n=16)$

duplicates $(n=7)$

not gout $(n=4)$

Figure 1 Selection of studies included in the review. CKD, chronic kidney disease. 
Table 1 Characteristics of included studies

\begin{tabular}{|c|c|c|c|c|c|c|c|c|c|}
\hline First author & Study period & Location & $\begin{array}{l}\text { Source of data/ } \\
\text { study population }\end{array}$ & Study type & Total sample & Mean age $(S D)^{a}$ & $\%$ male & Gout ascertainment & $\begin{array}{l}\text { Outcomes reported (method } \\
\text { of ascertainment) }\end{array}$ \\
\hline Chen [22] & 2006 & China & $\begin{array}{l}\text { Residents of } \\
\text { Changning district of } \\
\text { Shanghai }\end{array}$ & CS & 2,554 & 58.4 (SE 15.3) & 36 & Self-report & $\begin{array}{l}\text { Adjusted ORs for CKD any stage } \\
\text { and CKD stage } \geq 3 \text { (biochemical } \\
\text { measurement) }\end{array}$ \\
\hline \multirow[t]{2}{*}{ Hsu [23] } & \multirow[t]{2}{*}{1964 to 2000} & \multirow[t]{2}{*}{ USA } & \multirow{2}{*}{$\begin{array}{l}\text { Kaiser Permanente } \\
\text { Northern California } \\
\text { linked to US Renal } \\
\text { Data System }\end{array}$} & \multirow[t]{2}{*}{ Cohort } & \multirow[t]{2}{*}{177,570} & ESRD 42.3 (10.3) & ESRD 59 & \multirow{2}{*}{$\begin{array}{l}\text { Self-reported } \\
\text { physician diagnosis }\end{array}$} & \multirow{2}{*}{$\begin{array}{l}\text { Univariate and multivariate HRs } \\
\text { for ESRD requiring RRT (record } \\
\text { linkage) }\end{array}$} \\
\hline & & & & & & No ESRD 40.7 (14.0) & No ESRD 46 & & \\
\hline \multirow[t]{2}{*}{ Kuo [24] } & \multirow[t]{2}{*}{2000 to 2006} & \multirow[t]{2}{*}{ Taiwan } & \multirow{2}{*}{$\begin{array}{l}\text { Chang Gung } \\
\text { Memorial Hospital } \\
\text { health screening } \\
\text { programme }\end{array}$} & \multirow{2}{*}{$\begin{array}{l}\text { CS data from } \\
\text { cohort }^{\text {b }}\end{array}$} & \multirow[t]{2}{*}{61,527} & Males 49.1 (11.0) & 56 & \multirow{2}{*}{$\begin{array}{l}\text { Self-reported or ICD } \\
\text { code or crystal } \\
\text { identification }\end{array}$} & \multirow{2}{*}{$\begin{array}{l}\text { Prevalence of CKD stage } \geq 3 \\
\text { (biochemical measurement) }\end{array}$} \\
\hline & & & & & & Females 50.8 (10.8) & & & \\
\hline \multirow[t]{4}{*}{ Fuldeore [25] } & \multirow[t]{4}{*}{2002 to 2005} & \multirow[t]{4}{*}{ USA } & \multirow{4}{*}{$\begin{array}{l}\text { Managed Healthcare } \\
\text { Database }\end{array}$} & \multirow[t]{4}{*}{ CS } & \multirow[t]{4}{*}{3,929} & CKD & CKD & \multirow{4}{*}{$\begin{array}{l}\text { ICD code on two } \\
\text { occasions or ICD code } \\
\text { plus gout medication }\end{array}$} & \multirow{4}{*}{$\begin{array}{l}\text { Prevalence of CKD stage 2, } 3 \text { and } \\
4 \text { (biochemical measurement) }\end{array}$} \\
\hline & & & & & & 54.3 (NR) & 86 & & \\
\hline & & & & & & No CKD & No CKD & & \\
\hline & & & & & & $47.8(9.0)$ & 89 & & \\
\hline Johnson [26] & 1997 to 2004 & USA & $\begin{array}{l}\text { Kaiser Permanente } \\
\text { North West }\end{array}$ & CC & 5,335 & $64.4(\mathrm{NR})$ & 46 & $\begin{array}{l}\text { ICD code or SUA } \\
\geq 7 \mathrm{mg} / \mathrm{dl}\end{array}$ & $\begin{array}{l}\text { Unadjusted and adjusted ORs for } \\
\text { ESRD requiring RRT (medical } \\
\text { record review) }\end{array}$ \\
\hline Keenan [27] & 2007 to 2008 & USA & $\begin{array}{l}\text { New York Veterans } \\
\text { Affairs database }\end{array}$ & CS & 575 & $71.8(11.6)$ & 99 & ICD code & $\begin{array}{l}\text { Prevalence of } C K D \text { stage } \geq 3 \\
\text { (biochemical measurement or } \\
\text { ICD code) }\end{array}$ \\
\hline Yu [28] & 2000 to 2008 & Taiwan & $\begin{array}{l}\text { Taiwan National } \\
\text { Health Insurance } \\
\text { Database }\end{array}$ & Cohort & 656,108 & $41.1(15.6)$ & 73 & $\begin{array}{l}\text { ICD code on two } \\
\text { occasions or ICD code } \\
\text { plus gout medication }\end{array}$ & $\begin{array}{l}\text { Multivariate HR for ESRD } \\
\text { requiring RRT (record linkage) }\end{array}$ \\
\hline O'Sullivan [34] & 1964 & USA & $\begin{array}{l}\text { Population of } \\
\text { Sudbury, } \\
\text { Massachusetts }\end{array}$ & CS & 4,626 & NR & NR & $\begin{array}{l}\text { New York/Rome } \\
\text { criteria }\end{array}$ & $\begin{array}{l}\text { Prevalence of nephrolithiasis } \\
\text { (self-report, not defined) }\end{array}$ \\
\hline $\begin{array}{l}\text { Schaffalitzky De } \\
\text { Muckadell [35] }\end{array}$ & 1973 & Denmark & $\begin{array}{l}\text { Male office workers } \\
\text { aged } 40 \text { to } 59 \text { years in } \\
\text { Copenhagen }\end{array}$ & CS & 312 & NR & 100 & $\begin{array}{l}\text { Self-reported } \\
\text { physician diagnosis }\end{array}$ & $\begin{array}{l}\text { Prevalence of nephrolithiasis } \\
\text { (self-report, not defined) }\end{array}$ \\
\hline Currie [36] & 1969 to 1975 & UK & $\begin{array}{l}\text { General practice } \\
\text { records }\end{array}$ & CS & 604 & 52.3 (NR) & 77 & GP diagnosis & $\begin{array}{l}\text { Prevalence of nephrolithiasis (GP } \\
\text { diagnosis) }\end{array}$ \\
\hline Currie [37] & 1976 & UK & $\begin{array}{l}\text { General practice } \\
\text { records }\end{array}$ & CS & 64,454 & $N R$ & NR & GP diagnosis & $\begin{array}{l}\text { Incidence of nephrolithiasis (GP } \\
\text { diagnosis) }\end{array}$ \\
\hline \multirow[t]{2}{*}{ Kramer [38] } & \multirow[t]{2}{*}{1988 to 1994} & \multirow[t]{2}{*}{ USA } & \multirow{2}{*}{$\begin{array}{l}\text { National Health and } \\
\text { Nutrition Examination } \\
\text { Survey (NHANES) III }\end{array}$} & \multirow[t]{2}{*}{ CS } & \multirow[t]{2}{*}{17,030} & Stones $53.7(0.7)$ & 48 & \multirow{2}{*}{$\begin{array}{l}\text { Self-reported } \\
\text { physician diagnosis }\end{array}$} & \multirow{2}{*}{$\begin{array}{l}\text { Prevalence and adjusted ORs for } \\
\text { nephrolithiasis (self-reported } \\
\text { lifetime prevalence) }\end{array}$} \\
\hline & & & & & & No stones $44.2(0.4)$ & & & \\
\hline \multirow[t]{2}{*}{ Kramer [39] } & \multirow[t]{2}{*}{1986 to 1998} & USA & Health Professionals & Cohort & 51,529 & Gout 59.9 (9.1) & 100 & Self-reported & Prevalence and adjusted RRs for \\
\hline & & & & & & No gout 54.4 (9.8) & & $\begin{array}{l}\text { Pnysiclan alagnosis, } \\
\text { ARA criteria }\end{array}$ & $\begin{array}{l}\text { lifetime prevalence) } \\
\text { lifired }\end{array}$ \\
\hline
\end{tabular}


Table 1 Characteristics of included studies (Continued)

\begin{tabular}{|c|c|c|c|c|c|c|c|c|c|}
\hline \multirow[t]{2}{*}{ Mikuls [40] } & \multirow[t]{2}{*}{1990 to 1999} & \multirow[t]{2}{*}{ UK } & \multirow{2}{*}{$\begin{array}{l}\text { General Practice } \\
\text { Research Database } \\
\text { (GPRD) }\end{array}$} & \multirow[t]{2}{*}{ CS } & \multirow[t]{2}{*}{207,350} & Gout $60.5(15.4)$ & \multirow[t]{2}{*}{46} & \multirow[t]{2}{*}{ GP diagnosis } & \multirow{2}{*}{$\begin{array}{l}\text { Prevalence, unadjusted and } \\
\text { adjusted ORs for nephrolithiasis } \\
\text { (medical records) }\end{array}$} \\
\hline & & & & & & OA controls 66.8 (13.4) & & & \\
\hline \multirow[t]{2}{*}{ Harrold [41] } & \multirow[t]{2}{*}{1999 to 2003} & \multirow[t]{2}{*}{ USA } & \multirow{2}{*}{$\begin{array}{l}\text { Health Maintenance } \\
\text { Organisations (HMO) } \\
\text { Research Network } \\
\text { Centre for Education } \\
\text { and Research on } \\
\text { Therapeutics (CERT) }\end{array}$} & \multirow[t]{2}{*}{ CS } & \multirow[t]{2}{*}{6,133} & Males 58 (14) & \multirow[t]{2}{*}{81} & \multirow{2}{*}{$\begin{array}{l}\text { ICD code on two } \\
\text { occasions }\end{array}$} & \multirow{2}{*}{$\begin{array}{l}\text { Prevalence of nephrolithiasis } \\
\text { (ICD code) }\end{array}$} \\
\hline & & & & & & Females 70 (12) & & & \\
\hline Padang [42] & Not stated & Indonesia & $\begin{array}{l}\text { Community Health } \\
\text { Centres in Northern } \\
\text { Sulawesi }\end{array}$ & CS & 380 & NR & NR & $\begin{array}{l}\geq 3 \text { attacks of gout/ } \\
\text { year and/or tophi plus } \\
\text { ARA criteria }\end{array}$ & $\begin{array}{l}\text { Prevalence of nephrolithiasis } \\
\text { (self-report, not defined) }\end{array}$ \\
\hline Sarawate [43] & 1999 to 2004 & USA & $\begin{array}{l}\text { Managed Care } \\
\text { Database }\end{array}$ & $\begin{array}{l}\text { CS data from } \\
\text { cohort }^{\mathrm{b}}\end{array}$ & 5,942 & $57.4(14.1)$ & 76 & $\begin{array}{l}\text { ICD code on two } \\
\text { occasions or ICD code } \\
\text { plus gout medication }\end{array}$ & $\begin{array}{l}\text { Prevalence of nephrolithiasis } \\
\text { (ICD code) }\end{array}$ \\
\hline \multirow[t]{2}{*}{ Solomon [44] } & \multirow[t]{2}{*}{ Not stated } & \multirow[t]{2}{*}{ USA } & \multirow{2}{*}{$\begin{array}{l}\text { US Medicare system } \\
\text { and Pharmacy } \\
\text { Assistance Contract } \\
\text { for the Elderly (PACE) }\end{array}$} & \multirow{2}{*}{$\begin{array}{l}\text { CS data from } \\
\text { cohort }^{\mathrm{b}}\end{array}$} & \multirow[t]{2}{*}{9,823} & Male 78 (7) & \multirow[t]{2}{*}{16} & \multirow[t]{2}{*}{ Use of ULT } & \multirow{2}{*}{$\begin{array}{l}\text { Prevalence of nephrolithiasis } \\
\text { (medical records) }\end{array}$} \\
\hline & & & & & & Female $80(7)$ & & & \\
\hline Harrold [45] & 2000 to 2006 & USA & $\begin{array}{l}\text { Health Maintenance } \\
\text { Organisations (HMO) } \\
\text { Research Network } \\
\text { Centre for Education } \\
\text { and Research on } \\
\text { Therapeutics (CERT) }\end{array}$ & $\begin{array}{l}\text { CS data from } \\
\text { cohort }^{\mathrm{b}}\end{array}$ & 4,166 & $62(14)$ & 75 & $\begin{array}{l}\text { ICD code plus use of } \\
\text { ULT }\end{array}$ & $\begin{array}{l}\text { Prevalence of nephrolithiasis } \\
\text { (ICD code) }\end{array}$ \\
\hline \multirow[t]{2}{*}{ Roddy [49] } & \multirow[t]{2}{*}{2005} & \multirow[t]{2}{*}{ UK } & \multirow{2}{*}{$\begin{array}{l}\text { Registered population } \\
\text { of two general } \\
\text { practices in } \\
\text { Nottingham }\end{array}$} & \multirow[t]{2}{*}{ CS } & \multirow[t]{2}{*}{3,082} & Gout 63.8 (10.6) & Gout 81 & \multirow{2}{*}{$\begin{array}{l}\text { Self-reported gout, } \\
\text { validated by } \\
\text { rheumatologist }\end{array}$} & \multirow{2}{*}{$\begin{array}{l}\text { Prevalence of CKD stage } \geq 3, \geq 4 \\
\text { (biochemical measurement) and } \\
\text { nephrolithiasis (self-reported } \\
\text { lifetime prevalence) }\end{array}$} \\
\hline & & & & & & No gout 57.0 (14.4) & No gout 41 & & \\
\hline Zhu [48] & 2007 to 2008 & USA & $\begin{array}{l}\text { National Health and } \\
\text { Nutrition Examination } \\
\text { Survey (NHANES) }\end{array}$ & CS & 5,707 & 47 (NR) & 48 & $\begin{array}{l}\text { Self-reported } \\
\text { physician diagnosis }\end{array}$ & $\begin{array}{l}\text { Prevalence and adjusted OR for } \\
\text { CKD stage } \geq 2, \geq 3^{C} \text { (biochemical } \\
\text { measurement) and } \\
\text { nephrolithiasis (self-reported } \\
\text { lifetime prevalence) }\end{array}$ \\
\hline Liote [29] & 2008 to 2009 & France & $\begin{array}{l}\text { General Practitioner } \\
\text { data from GOSPEL } \\
\text { survey }\end{array}$ & CS & 810 & $62.7(11.3)$ & 87.2 & Physician diagnosis & $\begin{array}{l}\text { Prevalence of CKD stage } \geq 3, \geq 4 \\
\text { (biochemical measurement) }\end{array}$ \\
\hline Lin [30] & 2007 & Taiwan & $\begin{array}{l}\text { Community-based } \\
\text { survey, sampling via } \\
\text { Household Register } \\
\text { Database }\end{array}$ & CS & 3,352 & $47.5(17.4)$ & 48.6 & $\begin{array}{l}\text { Self-reported } \\
\text { physician diagnosis of } \\
\text { gout or } \\
\text { hyperuricaemia }\end{array}$ & $\begin{array}{l}\text { Prevalence of CKD stage } \geq 3 \\
\text { (biochemical measurement) }\end{array}$ \\
\hline Kuo [31] & 2000 to 2008 & Taiwan & $\begin{array}{l}\text { Longitudinal Health } \\
\text { Insurance Database }\end{array}$ & $\begin{array}{l}\text { CS data from } \\
\text { cohort }^{b}\end{array}$ & 704,503 & $42.73(16.6)$ & $\begin{array}{l}\text { Gout } 70.3 \\
\text { no gout } 50.4\end{array}$ & ICD code & $\begin{array}{l}\text { Prevalence and unadjusted OR } \\
\text { for ESRD requiring RRT (record } \\
\text { linkage) }\end{array}$ \\
\hline
\end{tabular}


Table 1 Characteristics of included studies (Continued)

\begin{tabular}{|c|c|c|c|c|c|c|c|c|c|}
\hline Krishnan [32] & 2009 to 2010 & USA & $\begin{array}{l}\text { National Health and } \\
\text { Nutrition Examination } \\
\text { Survey }\end{array}$ & CS & 5,589 & $44(21)$ & 49 & $\begin{array}{l}\text { Self-reported } \\
\text { physician diagnosis }\end{array}$ & $\begin{array}{l}\text { Prevalence of CKD stage 2, 3, } \\
\geq 4 \text { (biochemical measurement) }\end{array}$ \\
\hline Kok [33] & 2003 to 2007 & Taiwan & $\begin{array}{l}\text { Taiwan National } \\
\text { Health Insurance } \\
\text { Database }\end{array}$ & $\begin{array}{l}\text { CS data from } \\
\text { cohort }^{\mathrm{b}}\end{array}$ & $3,858,840$ & NR & 48.3 & $\begin{array}{l}\text { ICD code on three } \\
\text { occasions }\end{array}$ & $\begin{array}{l}\text { Prevalence of CKD, stages not } \\
\text { specified (ICD code) }\end{array}$ \\
\hline Trifiro [46] & 2005 to 2009 & Italy & $\begin{array}{l}\text { Health Search/ } \\
\text { Cegedim Strategic } \\
\text { Data Longitudinal } \\
\text { Patient Database }\end{array}$ & CS & 12,276 & NR & 74 & $\begin{array}{l}\text { ICD code, related } \\
\text { keywords for free text } \\
\text { search }\end{array}$ & $\begin{array}{l}\text { Prevalence and unadjusted OR } \\
\text { for nephrolithiasis (medical } \\
\text { records) }\end{array}$ \\
\hline Scales [13] & 2007 to 2010 & USA & $\begin{array}{l}\text { National Health and } \\
\text { Nutrition Examination } \\
\text { Survey }\end{array}$ & CS & 12,110 & NR & NR & NR & $\begin{array}{l}\text { Adjusted OR for nephrolithiasis } \\
\text { (self-reported lifetime prevalence) }\end{array}$ \\
\hline Ando [47] & 1995 to 2001 & Japan & $\begin{array}{l}\text { Men undergoing } \\
\text { medical examination } \\
\text { at Gifu Prefectural } \\
\text { Center for Health } \\
\text { Check and Health } \\
\text { Promotion }\end{array}$ & CS & 13,418 & $\begin{array}{l}\text { Controls } 48.5(8.8), \\
\text { past stones } 49.7(8.4)\end{array}$ & 100 & $\begin{array}{l}\text { Receiving medical } \\
\text { treatment for gout or } \\
\text { SUA } \geq 7.0 \mathrm{mg} / \mathrm{dl}\end{array}$ & $\begin{array}{l}\text { Prevalence nephrolithiasis } \\
\text { (self-reported lifetime prevalence) }\end{array}$ \\
\hline
\end{tabular}

ARA, American Rheumatism Association; CC, case-control study; CKD, chronic kidney disease; CS, cross-sectional; ESRD, end-stage renal disease; GP, general practitioner; HR, hazard ratio; ICD, International Classification of Disease; NR, not reported; OA, osteoarthritis; OR, odds ratio; RR, relative risk; RRT, renal replacement therapy; SD, standard deviation; SE, standard error; SUA, serum uric acid level; ULT, urate-lowering therapy.

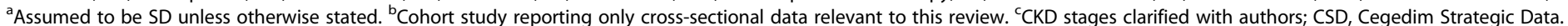


managed care setting used less than $70 \%$ of records available to them $[24,31]$.

Of the three cohort studies, none reported rates of attrition and one did not state whether the outcome (ESRD) was absent at the start of the study [23]. Length of follow-up was deemed adequate to determine outcome in all three studies.

\section{Selection for meta-analysis}

Seventeen out of 29 studies were included in the metaanalysis [13,22-24,28-30,32,34,35,38,39,42,46-49]. Two studies examining CKD were deemed unsuitable for pooling due to use of diagnostic codes [27,33] rather than biochemical testing to ascertain CKD that has been shown to significantly underestimate prevalence $[53,54]$. One study demonstrated significant incompleteness of biochemical data and was not pooled [25]. Ten out of 17 studies examining self-reported nephrolithiasis were pooled [13,34,35,38,39,42,46-49], and six studies were deemed too methodologically different to pool due to using a general practitioner completed questionnaire [36] or diagnostic codes and/or a limited time period for ascertaining nephrolithiasis [40,41,43-45]. Three studies reported outcomes that were not reported by other studies, thus precluding meta-analysis [26,31,37].

\section{Prevalence and risk of chronic kidney disease in gout}

Six studies provided suitable data to allow the pooled prevalence of CKD stage $\geq 3$ in people with gout to be calculated $[24,29,30,32,48,49]$. Further data $[29,30]$ and outcome clarification [48] was requested from the authors of three of these studies, enabling their inclusion. No reply was received from the authors of a further paper from whom the prevalence of CKD in those with and without gout was requested. The pooled prevalence estimate of CKD stage $\geq 3$ in people with gout was $24 \%$ (95\% CI 19\%, 28\%) (Figure 2). Statistically significant heterogeneity was identified $\left(I^{2}=84.3 \%, P<0.001\right)$. Data from three studies $[29,32,49]$ were pooled providing a prevalence estimate of CKD stage $\geq 4$ in gout of $2 \%(95 \%$ CI $0 \%$, 4\%) $\left(I^{2}=82.5 \%, P=0.003\right)$.

Comparing patients with gout to those without gout, the pooled unadjusted odds ratio (OR) between gout and CKD stage $\geq 3$ was 4.32 (95\% CI 3.82, 4.89) (Figure 3) based on the findings of three studies $[24,30,48]$. Pooling an age-adjusted and gender-adjusted OR and two multivariate ORs (adjustment included age, gender, obesity, hypertension, and diabetes mellitus) between gout and CKD stage $\geq 3$ resulted in an adjusted OR of 2.41 (95\% CI $1.86,3.11$ ) (Figure 3) $[22,30,48]$. There was no significant heterogeneity identified for either pooled unadjusted or adjusted ORs (unadjusted $I^{2}=0.0 \%, P=0.803$; adjusted $\left.I^{2}=0.0 \%, P=0.416\right)$.

One study reported prevalence of ESRD requiring RRT in gout $(0.6 \%)$ and provided an unadjusted OR for ESRD in gout versus no gout (OR 2.65; 95\% CI 2.25, 3.12) [31]. One retrospective matched case-control study compared the likelihood of a prior combined exposure of gout or

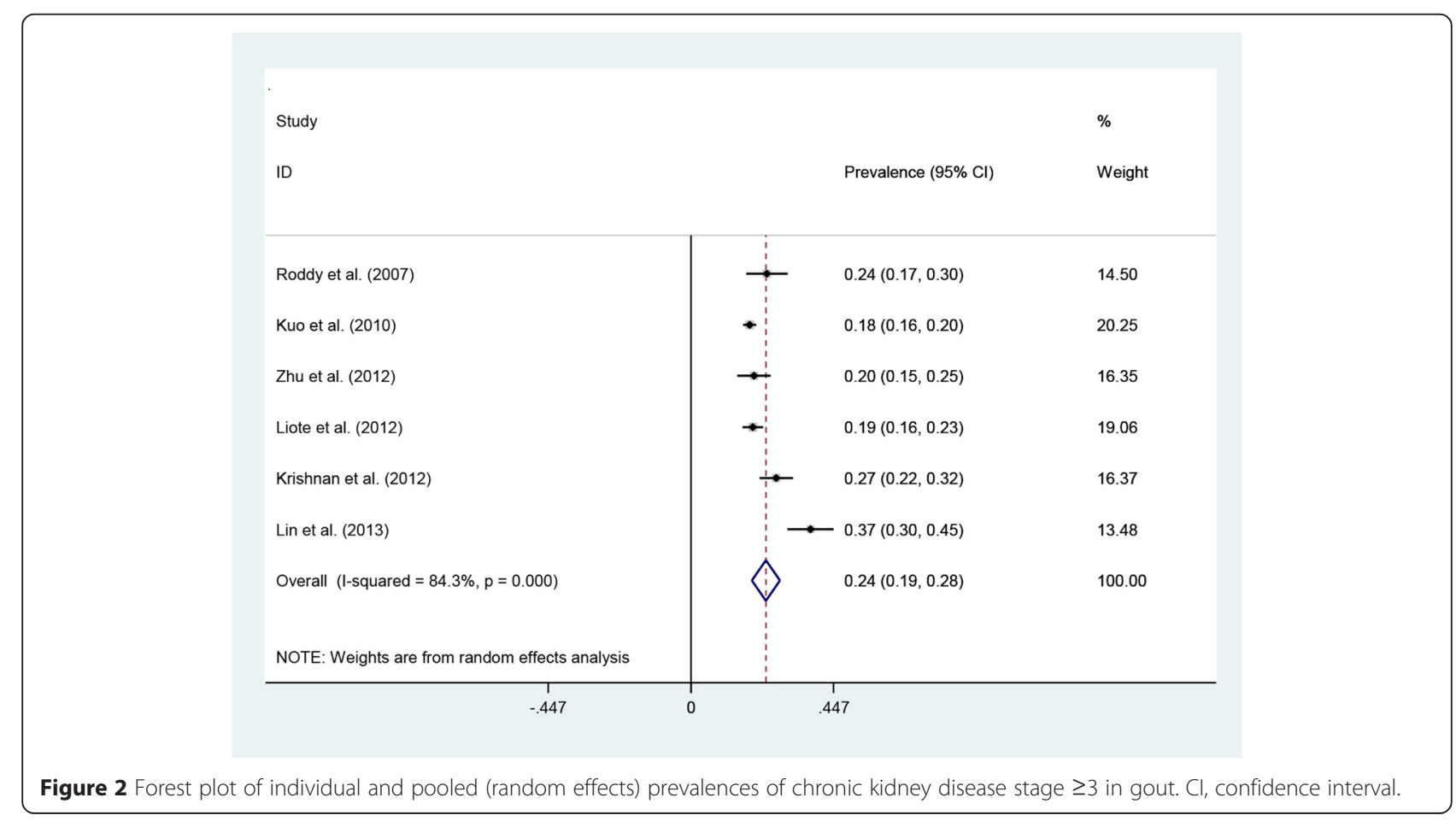




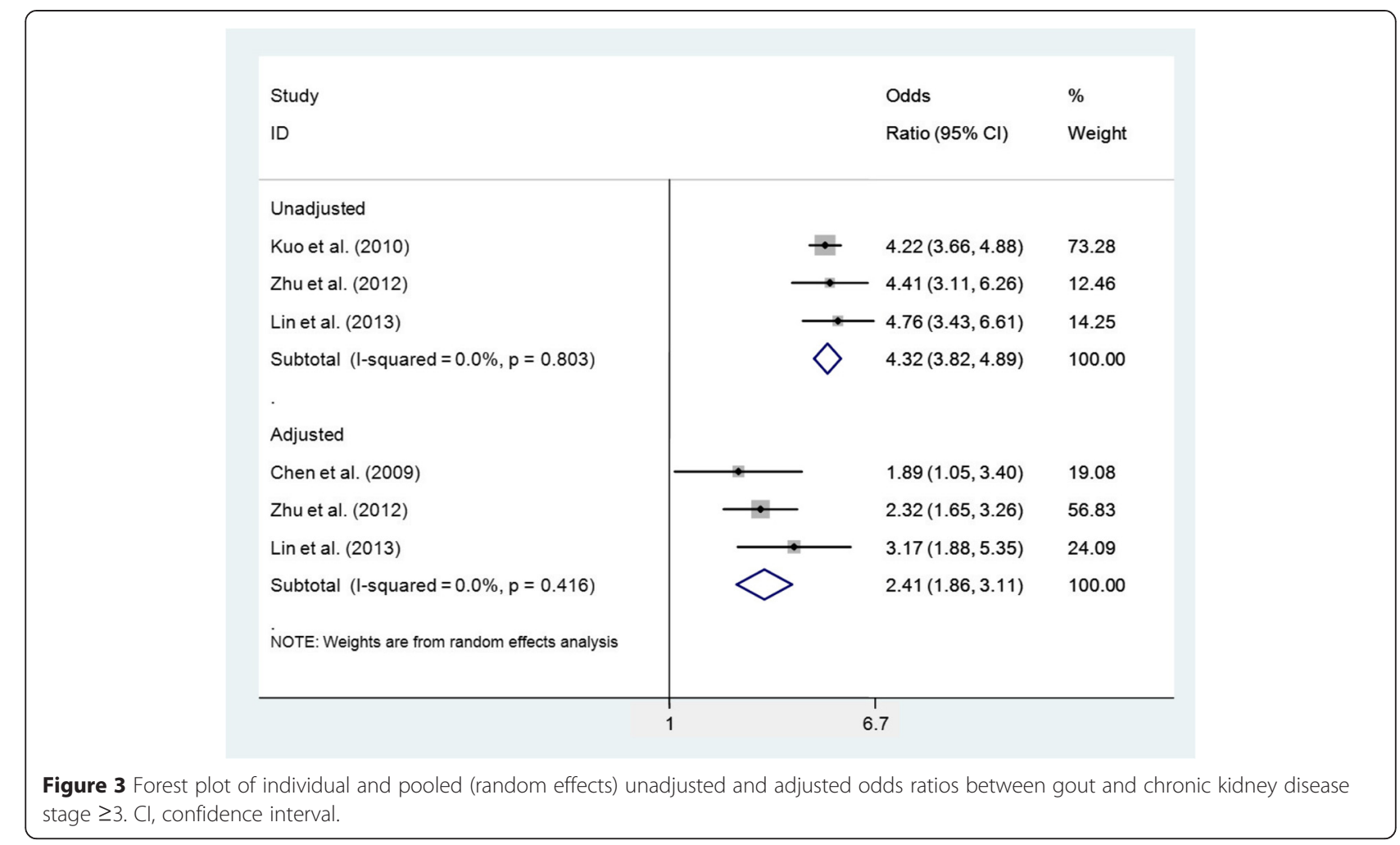

hyperuricaemia between cases with ESRD requiring RRT and controls without ESRD matched for age, gender, and year of RRT (adjusted OR 2.51; 95\% CI 1.78, 3.54) [26].

Following contact with the authors of one study to provide an unadjusted hazard ratio (HR) $(95 \% \mathrm{CI})$ between gout and ESRD [28], there were two prospective cohort studies $[23,28]$ that provided suitable data to allow a pooled unadjusted HR between gout and incident ESRD to be calculated (HR 2.69; 95\% CI 1.00, 4.39). Mean follow-up periods were 24.5 years in one study [23] and 8.0 years overall in the other [28]. Significant heterogeneity was identified $\left(I^{2} 93.1 \%, P<0.001\right)$. We contacted the authors of one of these studies to request an adjusted HR (95\% CI) between gout and incident ESRD [23]. The authors replied but were unable to provide this information. Only one study provided a multivariate adjusted HR between gout and incident ESRD (HR 1.57; 95\% CI 1.38 to 1.79 ; adjusted for age, sex, diabetes and hypertension) [28].

\section{Prevalence and risk of nephrolithiasis in gout}

We were able to use additional unpublished data from one of our previous studies [49] concerning the prevalence of self-reported lifetime nephrolithiasis in people with gout and ORs (95\% CI) between gout and self-reported lifetime nephrolithiasis, unadjusted and then adjusted, firstly, for age and gender and, secondly, for age, gender, diabetes, hypertension, and diuretic use. The pooled estimate of the prevalence of nephrolithiasis in people with gout was $14 \%$ (95\% CI 12\%, 17\%) (Figure 4) based on the findings of eight studies $[34,35,38,39,42,47-49]$. Significant statistical heterogeneity was identified $\left(I^{2}=79.4 \%, P<0.001\right)$.

Eight studies [34,35,38,39,42,46,48,49] provided suitable data to calculate a pooled unadjusted OR between gout and self-reported prevalence of nephrolithiasis (OR 2.66; 95\% CI 2.16, 3.27) (Figure 5). The pooled ageadjusted and gender-adjusted OR was 1.87 (95\% CI 1.25, 2.80 ), based on the findings of four studies [42,47-49]. Three studies provided suitable data to permit a pooled multivariate adjusted OR to be calculated (OR 1.77; 95\% CI 1.43, 2.19) $[13,38,49]$. All three studies adjusted for age, gender, and body mass index; two studies additionally adjusted for hypertension $[38,49]$ and the other study for diabetes mellitus [13]. Significant heterogeneity was identified for the pooled unadjusted and the age-adjusted and gender-adjusted ORs (unadjusted $I^{2}=69.1 \%, P=0.002$; age-adjusted and gender-adjusted $I^{2}=70.2 \%, P=0.018$ ) but not for the multivariate pooled estimate $\left(I^{2}=0.0 \%\right.$, $P=0.510)$. One prospective study found men with gout to have a relative risk of 2.12 (95\% CI 1.22, 3.68) of developing incident nephrolithiasis over a 12 -year period compared with men without gout, after adjustment for age, body mass index, diuretic use, hypertension, and dietary factors [39]. 

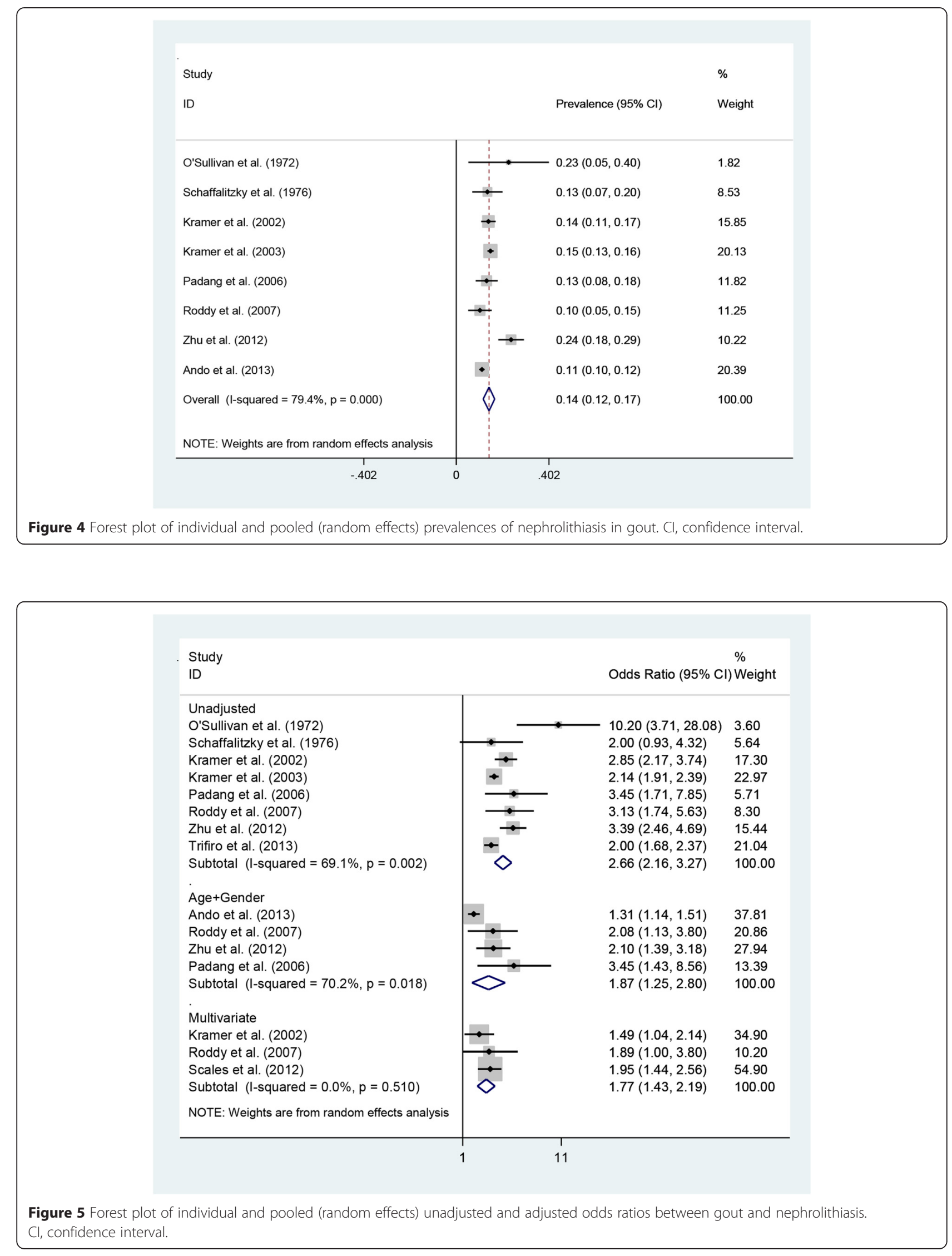


\section{Assessment of publication bias}

When pooling the six studies describing the prevalences of CKD stage $\geq 3$, Egger's test yielded a borderline result of $P=0.05$. No evidence of small study effects was seen for nephrolithiasis (Egger's test $P=0.548$ ).

\section{Discussion}

In this systematic review and meta-analysis of populationbased epidemiological studies, renal disease and nephrolithiasis were common findings in people with gout. The pooled prevalence estimates of CKD stage $\geq 3$ and selfreported nephrolithiasis in people with gout were $24 \%$ and $14 \%$ respectively. Furthermore, after adjustment for confounding variables, people with gout were more than twice as likely to have CKD stage $\geq 3$ (OR 2.41) and over one and a half times (OR 1.77) as likely to have ever had nephrolithiasis as people who did not have gout. Most studies employed a cross-sectional design and there was a paucity of prospective studies. The pooled unadjusted HR for incident ESRD in people with gout was 2.69; however, the lower limit of the 95\% CI bordered unity. Only one study provided an adjusted HR which suggested that gout is an independent risk factor for incident ESRD (HR 1.57). Similarly, the single prospective study of the risk of developing nephrolithiasis found people with gout to have twice the risk (adjusted relative risk 2.12) of people without gout.

To our knowledge, this is the first meta-analysis of associations between gout and CKD/nephrolithiasis. Strengths of our methodology include the comprehensive search strategy and literature review process undertaken by two independent reviewers, translation of non-English-language publications, contacting study authors to request additional data to maximise the number of included studies, and an outcome which required biochemically defined CKD rather than more heterogeneous and less precise clinical diagnostic labels.

As with any systematic review, our findings are dependent upon the size and quality of the published literature. There was only a small number of studies that had examined either CKD or nephrolithiasis. Studies of CKD were all published from 2007 onwards, which is not surprising as the National Kidney Foundation CKD classification was only introduced in 2002 [19]. Widening the outcome definition to include clinical diagnostic labels for kidney disease would have increased the number of available studies but would have increased heterogeneity. Furthermore, CKD classification is widely used and highly relevant to clinical practice. The small number of published studies also raises the possibility of publication bias. Although not evident for nephrolithiasis, there were too few studies of CKD to examine for publication bias. There were several methodological limitations of the included studies worthy of further discussion. The majority of included studies were not designed with the primary aim of assessing the association between gout and $\mathrm{CKD} /$ nephrolithiasis. Most studies based the diagnosis of gout on physician diagnosis (which has been shown to be reasonably accurate [49]) or patient self-report rather than more rigorous methods such as the American Rheumatism Association criteria [50] or crystal identification. This limitation risks misclassification bias but, as highlighted by this study, the current evidence base offers limited numbers of epidemiological studies using more robust methods. Several studies did not include control groups without gout and only reported the prevalence of CKD/ nephrolithiasis in gout but not the strength of association between the two. Although our findings demonstrate a clear independent association between gout and both CKD and nephrolithiasis, there were few prospective studies so we could not draw firm conclusions about temporal aspects of these associations. Previous epidemiological studies have shown that chronic renal disease is an independent risk factor for gout $[10,11]$, yet there are several plausible mechanisms by which gout might predispose to CKD. Renal damage can result from co-morbid hypertension and diabetes, hyperuricaemiamediated endothelial dysfunction and renovascular disease [55], and use of nonsteroidal anti-inflammatory drugs. Although allopurinol is widely believed to have a deleterious effect on renal function based on early observations [56], a recent systematic review suggested that it may protect against progression of CKD [57]. Inflammation in gout is increasingly recognised to persist in the intercritical period between acute attacks [58,59], raising the possibility that inflammatory mechanisms contribute to vascular risk, as has been proposed for other inflammatory arthropathies [60]. Statistical heterogeneity was demonstrated in the meta-analyses for CKD and nephrolithiasis prevalence, the unadjusted HR between gout and incident ESRD, and the unadjusted and age-adjusted and genderadjusted ORs between gout and nephrolithiasis. Possible sources include differences between the demographic and co-morbid characteristics of the populations studied and the different follow-up periods in the prospective studies. It is noteworthy, however, that the pooled multivariate estimates adjusting for such demographic and co-morbid factors did not demonstrate significant heterogeneity.

\section{Conclusions}

The main clinical implications of our findings are that patients with gout should be screened for CKD and that clinicians should be made aware of the associations between gout and CKD/nephrolithiasis. Unless sought for, CKD usually progresses subclinically until reaching more advanced stages. In view of this, the significant morbidity and mortality associated with CKD [8] and the risk of CKD associated with gout, a presentation with gout in 
primary care should be viewed as a red flag for CKD and should prompt screening for and treatment of both CKD and its associated risk factors such as hypertension and diabetes mellitus, which are also risk factors for gout [10]. American College of Rheumatology guidelines advise considering CKD and nephrolithiasis as part of the management of gout [61] but current national and international guidelines regarding CKD [62] and nephrolithiasis [63] do not recognise gout as a risk factor for these conditions. Only one in five people presenting to primary care with acute gout are screened for CKD within a month of presentation [64].

In summary, gout is associated with both CKD and nephrolithiasis. However, there were insufficient prospective studies to determine the temporal nature of these associations or to determine the mechanisms underlying them. Whilst the associations seen suggest that clinicians should be made aware of them and that patients with gout should be screened for CKD, further studies are required to investigate these relationships further.

\section{Abbreviations}

Cl: confidence interval; CKD: chronic kidney disease; ESRD: end-stage renal disease; HR: hazard ratio; OR: odds ratio; RRT: renal replacement therapy.

\section{Competing interests}

All authors have completed the Unified Competing Interest form at www. icmje.org/coi_disclosure.pdf (available on request from the corresponding author) and declare that: the authors do not have any support from any company for the submitted work; the authors do not have any relationships with any companies that might have an interest in the submitted work in the previous 3 years; their spouses, partners, or children do not have any financial relationships that may be relevant to the submitted work; and the authors do not have any non-financial interests that may be relevant to the submitted work.

\section{Authors' contributions}

ER and CDM conceived the study. Analysis was undertaken by MJR and JB. All authors were involved in the design, interpretation of data, and drafting, revising and final approval of the manuscript. ER is guarantor and affirms that the manuscript is an honest, accurate and transparent account of the study being reported, and that no important aspects of the study have been omitted. There are no discrepancies from the study as planned. All authors had full access to all of the data (including statistical reports and tables) in the study and can take responsibility for the integrity of the data and the accuracy of the data analysis.

\section{Acknowledgements}

The authors would like to thank the authors of included studies who responded to queries enabling their studies to be included in this meta-analysis: Dr Chang-Fu Kuo, Professor Nan Chen, Dr Ming-Yen Lin, and Professor Frederic Liote for provision of additional data from their studies; Professor Hyon Choi, Professor Ryosuke Ando, and Dr Victor Kok for clarifying methodological aspects of their studies; and Professor Chi-Yuan Hsu for replying to our query and confirming that additional data were not available. The authors would also like to thank Professor Danielle van der Windt, Dr Nadia Corp, and Dr Olalekan Uthman for methodological advice and Professor Danielle van der Windt, Professor Victoria Barskova, Dr Kate Dunn, Simona Tchakarova, Dr Maryia Horava, and Kanchan Vohora for assistance with translation of non-English-language articles.

MJR received a bursary from the Jean Shanks Foundation to fund his intercalated MPhil. CDM is funded by the National Institute for Health Research (NIHR) Collaborations for Leadership in Applied Health Research and Care West Midlands, the NIHR School for Primary Care Research, and a NIHR Research Professorship in General Practice. This article presents independent research funded by the NIHR. The views expressed are those of the author(s) and not necessarily those of the National Health Service, the NIHR, or the Department of Health. The study funders had no role in the study design; the data collection, analysis, or interpretation; the writing of the paper; or the decision to submit the paper for publication.

\section{Author details}

${ }^{1}$ School of Medicine, Keele University, Keele, Staffordshire ST5 5BG, UK.

${ }^{2}$ School of Computing and Mathematics, Keele University, Keele, Staffordshire ST5 5BG, UK. ${ }^{3}$ Arthritis Research UK Primary Care Centre, Keele University, Keele, Staffordshire ST5 5BG, UK.

Received: 6 January 2015 Accepted: 24 March 2015

Published online: 01 April 2015

\section{References}

1. Kuo C, Grainge MJ, Mallen C, Zhang W, Doherty M. Rising burden of gout in the UK but continuing suboptimal management: a nationwide population study. Ann Rheum Dis. 2014;0:1-7.

2. Roddy E, Doherty M. Epidemiology of gout. Arthritis Res Ther. 2010;12:223.

3. Berger $L$, Yü TF. Renal function in gout. IV. An analysis of 524 gouty subjects including long-term follow-up studies. Am J Med. 1975;59:605-13.

4. Fessel WJ. Renal outcomes of gout and hyperuricemia. Am J Med. 1979;67:74-82.

5. Yü TF, Gutman AB. Uric acid nephrolithiasis in gout. Predisposing factors. Ann Intern Med. 1967:67:1133-48.

6. Yü TF, Berger L. Impaired renal function gout: its association with hypertensive vascular disease and intrinsic renal disease. Am J Med. 1982;72:95-100

7. Stevens PE, O'Donoghue DJ, de Lusignan S, Van Vlymen J, Klebe B, Middleton R, et al. Chronic kidney disease management in the United Kingdom: NEOERICA project results. Kidney Int. 2007;72:92-9.

8. Matsushita K, van der Velde M, Astor BC, Woodward M, Levey AS, de Jong $\mathrm{PE}$, et al. Association of estimated glomerular filtration rate and albuminuria with all-cause and cardiovascular mortality in general population cohorts: a collaborative meta-analysis. Lancet. 2010;375:2073-81.

9. Bello AK, Nwankwo E, El Nahas AM. Prevention of chronic kidney disease: a global challenge. Kidney Int Suppl. 2005;68:s11-7.

10. Choi HK, Atkinson K, Karlson EW, Curhan G. Obesity, weight change, hypertension, diuretic use, and risk of gout in men: the health professionals follow-up study. Arch Intern Med. 2005;165:742-8.

11. Cea Soriano L, Rothenbacher D, Choi HK, Garcia Rodriguez LA. Contemporary epidemiology of gout in the UK general population. Arthritis Res Ther. 2011;13:R39.

12. El-Zawawy H, Mandell BF. Managing gout: how is it different in patients with chronic kidney disease? Cleve Clin J Med. 2010;77:919-28.

13. Scales C, Smith A, Hanley J, Saigal C. Prevalence of kidney stones in the United States. Eur Urol. 2012;62:160-5.

14. Parmar MS. Kidney stones. BMJ. 2004;328:1420-4.

15. Liebman SE, Taylor JG, Bushinsky DA. Uric acid nephrolithiasis. Curr Rheumatol Rep. 2007;9:251-7.

16. Taylor EN, Stampfer MJ, Curhan GC. Diabetes mellitus and the risk of nephrolithiasis. Kidney Int. 2005;68:1230-5.

17. Taylor EN, Stampfer MJ, Curhan GC. Obesity, weight gain, and the risk of kidney stones. JAMA. 2005;293:455-62.

18. Cappuccio FP, Siani A, Barba G, Mellone MC, Russo L, Farinaro E, et al. A prospective study of hypertension and the incidence of kidney stones in men. J Hypertens. 1999;17:1017-22.

19. National Kidney Foundation. K/DOQI clinical practice guidelines for chronic kidney disease: evaluation, classification, and stratification. Am J Kidney Dis. 2002;39:51-266.

20. Wells G, Shea B, O'Connell D, Peterson J, Welch V, Losos M, et al. The Newcastle-Ottawa scale (NOS) for assessing the quality of nonrandomised studies in meta-analysis. http://www.ohri.ca/programs/_linical_epidemiology/ oxford.asp. Accessed 2 Dec 2011

21. Higgins JPT, Thompson SG, Deeks JJ, Altman DG. Measuring inconsistency in meta-analyses. BMJ. 2003;327:557-60.

22. Chen N, Wang W, Huang Y, Shen P, Pei D, Yu H, et al. Community-based study on CKD subjects and the associated risk factors. Nephrol Dial Transplant. 2009;24:2117-23. 
23. Hsu C, Iribarren C, McCulloch CE, Darbinian J, Go AS. Risk factors for end-stage renal disease - 25-year follow-up. Arch Intern Med. 2009;169:342-50.

24. Kuo CF, See LC, Luo SF, Ko YS, Lin YS, Hwang JS, et al. Gout: an independent risk factor for all-cause and cardiovascular mortality. Rheumatology (Oxford). 2010;49:141-6.

25. Fuldeore MJ, Riedel AA, Zarotsky V, Pandya BJ, Dabbous O, Krishnan E. Chronic kidney disease in gout in a managed care setting. BMC Nephrol. 2011;12:36.

26. Johnson ES, Smith DH, Thorp ML, Yang $X$, Juhaeri J. Predicting the risk of end-stage renal disease in the population-based setting: a retrospective case-control study. BMC Nephrol. 2011;12:17.

27. Keenan RT, O'Brien WR, Lee KH, Crittenden DB, Fisher MC, Goldfarb DS, et al, Prevalence of contraindications and prescription of pharmacologic therapies for gout. Am J Med. 2011;124:155-63.

28. Yu KH, Kuo CF, Luo SF, See LC, Chou IJ, Chang HC, et al. Risk of end-stage renal disease associated with gout: a nationwide population study. Arthritis Res Ther. 2012;14:R83.

29. Liote F, Lancrenon S, Lanz S, Guggenbuhl P, Lambert C, Saraux A, et al. GOSPEL: prospective survey of gout in France. Part l: design and patient characteristics ( $n=1003$ ). Joint Bone Spine. 2012;79:464-70.

30. Lin MY, Chiu YW, Lee CH, Yu HY, Chen HC, Wu MT, et al. Factors associated with CKD in the elderly and nonelderly population. Clin J Am Soc Nephrol. 2013;8:33-40.

31. Kuo CF, Yu KH, See LC, Chou IJ, Ko YS, Chang HC, et al. Risk of myocardial infarction among patients with gout: a nationwide population-based study. Rheumatology (Oxford). 2013;52:111-7.

32. Krishnan E. Reduced glomerular function and prevalence of gout: NHANES 2009-10. PLoS One. 2012;7:e50046.

33. Kok VC, Horng JT, Lin HL, Chen YC, Chen YJ, Cheng KF. Gout and subsequent increased risk of cardiovascular mortality in non-diabetics aged 50 and above: a population-based cohort study in Taiwan. BMC Cardiovasc Disord. 2012;12:108.

34. O'Sullivan JB. Gout in a New England town. A prevalence study in Sudbury, Massachusetts. Ann Rheum Dis. 1972;31:166-9.

35. Schaffalitzky De Muckadell OB, Gyntelberg F. Occurrence of gout in Copenhagen males aged 40-59. Int J Epidemiol. 1976:5:153-8.

36. Currie WJ. The gout patient in general practice. Rheumatol Rehabil. 1978;17:205-17.

37. Currie WJ, Turmer P. The frequency of renal stones within Great Britain in a gouty and non-gouty population. Br J Urol. 1979:51:337-41.

38. Kramer HM, Curhan G. The association between gout and nephrolithiasis: The National Health and Nutrition Examination Survey III, 1988-1994. Am J Kidney Dis. 2002;40:37-42.

39. Kramer HJ, Choi HK, Atkinson K, Stampfer M, Curhan GC. The association between gout and nephrolithiasis in men: The Health Professionals' Follow-Up Study. Kidney Int. 2003;64:1022-6.

40. Mikuls TR, Farrar JT, Bilker WB, Fernandes S, Schumacher Jr HR, Saag KG. Gout epidemiology: results from the UK general practice research database, 1990-1999. Ann Rheum Dis. 2005:64:267-72.

41. Harrold LR, Yood RA, Mikuls TR, Andrade SE, Davis J, Fuller J, et al. Sex differences in gout epidemiology: evaluation and treatment. Ann Rheum Dis. 2006;65:1368-72

42. Padang C, Muirden KD, Schumacher HR, Darmawan J, Nasution AR. Characteristics of chronic gout in Northern Sulawesi, Indonesia. J Rheumatol. 2006:33:1813-7.

43. Sarawate CA, Brewer KK, Yang W, Patel PA, Schumacher H, Saag KG, et al. Gout medication treatment patterns and adherence to standards of care from a managed care perspective. Mayo Clin Proc. 2006;81:925-34.

44. Solomon DH, Avorn J, Levin R, Brookhart MA. Uric acid lowering therapy: prescribing patterns in a large cohort of older adults. Ann Rheum Dis. 2008;67:609-13

45. Harrold LR, Andrade SE, Briesacher BA, Raebel MA, Fouayzi H, Yood RA, et al. Adherence with urate-lowering therapies for the treatment of gout. Arthritis Res Ther. 2009;11.

46. Trifiro G, Morabito P, Cavagna L, Ferrajolo C, Pecchioli S, Simonetti M, et al. Epidemiology of gout and hyperuricaemia in Italy during the years 2005-2009: a nationwide population-based study. Ann Rheum Dis. 2013;72:694-700.

47. Ando R, Nagaya T, Suzuki S, Takahashi H, Kawai M, Okada A, et al. Kidney stone formation is positively associated with conventional risk factors for coronary heart disease in Japanese men. J Urol. 2013:189:1340-6.
48. Zhu Y, Pandya BJ, Choi HK. Comorbidities of gout and hyperuricemia in the US general population: NHANES 2007-2008. Am J Med. 2012;125:679-687.e1.

49. Roddy E, Zhang W, Doherty M. Concordance of the management of chronic gout in a UK primary-care population with the EULAR gout recommendations. Ann Rheum Dis. 2007;66:1311-5.

50. Wallace SL, Robinson H, Masi AT, Decker JL, McCarty DJ, Yü TF. Preliminary criteria for the classification of the acute arthritis of primary gout. Arthritis Rheum. 1977;20:895-900.

51. Kellgren JH, Jeffrey MR, Ball J. The epidemiology of chronic rheumatism. Oxford: Blackwell; 1963

52. Bennett PH, Wood PHN. Population studies of the rheumatic diseases. Amsterdam: Excerpta Medica Foundation; 1968.

53. Glynn LG, Anderson J, Reddan D, Murphy AW. Chronic kidney disease in general practice: prevalence, diagnosis, and standards of care. Ir Med J. 2009;102:285-8.

54. de Lusignan S, Chan T, Stevens P, O'Donoghue D, Hague N, Dzregah B, et al. Identifying patients with chronic kidney disease from general practice computer records. Fam Pract. 2005;22:234-41.

55. Jin M, Yang F, Yang I, Yin Y, Luo JJ, Wang H, et al. Uric acid, hyperuricemia and vascular diseases. Front Biosci. 2012;17:656-69.

56. Rosenfeld JB. Effect of long-term allopurinol administration on serial GFR in normotensive and hypertensive hyperuricemic subjects. Adv Exp Med Biol. 1974;41:581-96.

57. Bose B, Badve SV, Hiremath SS, Boudville N, Brown FG, Cass A, et al. Effects of uric acid-lowering therapy on renal outcomes: a systematic review and meta-analysis. Nephrol Dial Transplant. 2014;29:406-13.

58. Roddy E, Menon A, Hall A, Datta P, Packham J. Polyarticular sonographic assessment of gout: a hospital-based cross-sectional study. Joint Bone Spine. 2013:80:295-300.

59. Pascual E. Persistence of monosodium urate crystals and low-grade inflammation in the synovial fluid of patients with untreated gout. Arthritis Rheum. 1991:34:141-5.

60. Soltész P, Kerekes G, Dér H, Szücs G, Szántó S, Kiss E, et al. Comparative assessment of vascular function in autoimmune rheumatic diseases: considerations of prevention and treatment. Autoimmun Rev. 2011;10:416-25.

61. Khanna D, Fitzgerald JD, Khanna PP, Bae S, Singh MK, Neogi T, et al. 2012 American College of Rheumatology guidelines for management of gout. Part 1: systematic nonpharmacologic and pharmacologic therapeutic approaches to hyperuricemia. Arthritis Care Res. 2012;64:1431-46.

62. National Institute for Health and Care Excellence. CG182 chronic kidney disease: early identification and management of chronic kidney disease in adults in primary and secondary care. London: National Institute for Health and Care Excellence; 2014.

63. Türk C, Knoll T, Petrik A, Sarica K, Skolarikos A, Straub M, et al. Guidelines on Urolithiasis. 2014. http://uroweb.org/guideline/urolithiasis/. Accessed 16 Mar 2015

64. Roddy E, Mallen CD, Hider SL, Jordan KP. Prescription and comorbidity screening following consultation for acute gout in primary care. Rheumatology (Oxford). 2010;49:105-11.

\section{Submit your next manuscript to BioMed Central and take full advantage of:}

- Convenient online submission

- Thorough peer review

- No space constraints or color figure charges

- Immediate publication on acceptance

- Inclusion in PubMed, CAS, Scopus and Google Scholar

- Research which is freely available for redistribution 